\title{
Stability Analysis of an Approximate Scheme for Moving Horizon Estimation*
}

\author{
Victor M. Zavala \\ Mathematics and Computer Science Division \\ Argonne National Laboratory, Argonne, IL 60439, USA
}

\begin{abstract}
We analyze the stability properties of an approximate algorithm for moving horizon estimation (MHE). The strategy provides instantaneous state estimates and is thus suitable for large-scale feedback control. In particular, we study the interplay between numerical approximation errors and the convergence of the estimator error. In addition, we establish connections between the numerical properties of the Hessian of the MHE problem and traditional observability definitions. We demonstrate the developments through a simulation case study.
\end{abstract}

Keywords: estimation, stability, large-scale, observability, nonlinear programming

\section{Introduction and Basic Notation}

In this paper, we consider the problem of state estimation for nonlinear discrete-time systems of the form

$$
\begin{aligned}
x_{t+1} & =f\left(x_{t}, u_{t}\right)+\xi_{t}, \quad t \geq 0 \\
y_{t} & =h\left(x_{t}\right)+\eta_{t}, \quad t \geq 0
\end{aligned}
$$

where $x_{t} \in \mathcal{X} \subseteq \Re^{n_{x}}$ is the state of the system, $u_{t} \in \mathcal{U} \subseteq \Re^{n_{u}}$ are the inputs, and $y_{t} \in \Re^{n_{y}}$ are the measured outputs. Symbols $\xi_{t} \in \Xi \subseteq \Re^{n_{\xi}}$ and $\eta_{t} \in \mathcal{H} \subseteq \Re^{n_{\eta}}$ denote bounded process and measurement noise disturbances, respectively. The nonlinear mappings $f: \Re^{n_{x}} \times \Re^{n_{u}} \rightarrow n_{x}$ and $h: \Re^{n_{x}} \rightarrow \Re^{n_{y}}$ represent the state and output models, respectively.

Moving horizon estimation (MHE) strategies use a moving measurement window,

$$
I_{t}^{T}=\left[I_{t}^{y T}, I_{t}^{u T}\right]=\left[y_{t-N}, \ldots, y_{t-1}, y_{t}, u_{t-N}, \ldots, u_{t-1}\right], t \geq 0
$$

to compute estimates $\hat{x}_{t}$ of the true state $x_{t}$. Here, $N$ is the size of the moving window, and $I_{t} \in \Re^{(N+1) n_{y}+N n_{u}}$ is the information vector at time $t$. The MHE formulation used in

\footnotetext{
${ }^{*}$ Preprint ANL/MCS-P1644-0609
} 
this work has the following form:

$$
\begin{array}{ll}
\min _{z_{0}} & J\left(z_{0}, \bar{x}_{t-N}^{o}, I_{t}\right):=\mu\left\|z_{0}-\bar{x}_{t-N}^{o}\right\|^{2}+\sum_{k=0}^{N}\left\|y_{t-N+k}-h\left(z_{k}\right)\right\|^{2} \\
\text { s.t. } & z_{k+1}=f\left(z_{k}, u_{t-N+k}\right), \quad k=0, \ldots, N-1 \\
& z_{k} \in \mathcal{X}, \quad k=0, \ldots, N,
\end{array}
$$

where the objective function (3a) incorporates the arrival cost and the least-squares output errors along the horizon, (3b) is the state model, and (3c) are the constraints. The optimal solution of this nonlinear programming problem (NLP) provides the state trajectory $\left[z_{0}^{o}, \ldots, z_{N}^{o}\right]$ from which we extract the initial state estimate $\hat{x}_{t-N}^{o} \leftarrow z_{0}^{o}$ and, implicitly, the current state $\hat{x}_{t}^{o} \leftarrow z_{N}^{o}$. In the following, we will use only the initial state estimate to refer to the solution of the MHE problem. Accordingly, the estimator error at the current time $t$ is defined as $e_{t-N}^{o}:=\hat{x}_{t-N}^{o}-x_{t-N}$. The optimal cost is denoted as $J\left(\hat{x}_{t-N}^{o}, \bar{x}_{t-N}^{o}, I_{t}\right)$. The symbol $\bar{x}_{t-N}^{o}$ denotes the reference or prior value of the initial state; $\mu$ is a regularization parameter fixed by design. At the next time step $t+1$, once we have the new measurements $y_{t+1}$ and $u_{t}$, we shift the measurement window forward: $I_{t+1}^{T}=\left[y_{t-N+1}, \ldots, y_{t}, y_{t+1}, u_{t-N+1}, \ldots, u_{t}\right]$. In addition, the reference initial state is updated as $\bar{x}_{t-N+1}^{o} \leftarrow f\left(\hat{x}_{t-N}^{o}, u_{t-N}\right)$, and the next MHE problem is solved to optimality. In the following, we will refer to the above strategy as the optimal MHE algorithm.

One of the main problems associated with MHE is to establish stability conditions for the estimation error $e_{t}:=\hat{x}_{t}-x_{t}, t \geq 0$. Different stability studies have been reported. In [16], the authors derive stability conditions for an estimator formulation assuming that the output errors vanish at the solution. With this, the estimator can be cast as a system of nonlinear equations, and stability properties can be established using fixed-point arguments. The analysis in [15] establishes stability by using Lyapunov arguments for an optimization-based estimator that penalizes only least-squares output errors. This work was extended in a comprehensive stability analysis presented in [19]. Here, the authors analyze the MHE problem as a forward dynamic programming approximation of the optimal batch estimator. Using this connection, they introduce the notion of the arrival cost and establish stability conditions using Lyapunov arguments. Their MHE strategy uses a more general least-squares objective than (3a), including prior and noise covariance matrices. This permits the authors to establish statistical properties of the estimator. In addition, the strategy computes estimates of the process noise sequences $\xi_{t}$ and handles constraints. The stability analysis in [2] uses the above MHE formulation (3). In this formulation, process noise $\xi_{t}$ is treated as a non estimable disturbance. In addition, the arrival cost uses a fixed prior diagonal matrix of the form $\mu \cdot \mathbb{I}_{n_{x}}, \mu \geq 0$. As expected, the stability and statistical properties of this estimator are less general compared to those in [19]. In particular, this formulation is entirely deterministic. Consequently, the least-squares objective is meaningless from a statistical point of view. These deterministic formulations have been studied in $[15,16,1]$ and they permit a more transparent analysis of the impact of the system properties on the stability of the estimator from which much insight can be obtained. Motivated by this, we use this formulation and associated stability arguments here. Probabilistic formulations and stability analyzes are presented in detail in [13]. 
A problem that arises in most practical MHE implementations is that the NLP (3) is a computationally intensive optimization problem. For instance, when MHE is used for feedback control, the solution time of the NLP introduces a nonnegligible feedback delay that deteriorates closed-loop performance [9]. In this work, we consider an approximate MHE formulation with minimal on-line computational effort and analyze its stability properties. The strategy is a simplification of the advanced-step MHE estimator presented in [24] in which nearly instantaneous approximate estimates are constructed by using reference solutions computed in background (i.e., between sampling times). We analyze the impact of numerical approximation errors on the stability properties of the algorithm and contrast these with those of the optimal MHE algorithm. In addition, we establish connections between the observability properties of the nonlinear system and the properties of the MHE problem. We illustrate the developments using a simulation case study.

The paper is structured as follows. In Section 2 we derive the approximate MHE algorithm. In Section 3 we establish system and observability properties. In Section 4 we derive stability conditions for the optimal and approximate MHE estimators. In Section 5 we present the numerical study. We close the paper with concluding remarks and directions of future work.

\section{Approximate MHE Algorithm}

To construct the approximate MHE algorithm, we recognize that, at time step $t-1$, we can use the current state estimate $\hat{x}_{t-1}^{\epsilon}$ and input $u_{t-1}$ to predict the future state and associate measurement $\bar{x}_{t}^{\epsilon}=f\left(\hat{x}_{t-1}^{\epsilon}, u_{t-1}\right)$ and $\bar{y}_{t}=h\left(\bar{x}_{t}^{\epsilon}\right)$, respectively. With these, we can use the predicted information vector $\bar{I}_{t}=\left[\bar{I}_{t}^{y}, I_{t}^{u}\right]^{T}=\left[y_{t-N}, \ldots, y_{t-1}, \bar{y}_{t}, u_{t-N}, \ldots, u_{t-1}\right]^{T}$ to solve, between sampling times, the background MHE problem:

$$
\begin{array}{ll}
\min _{z_{0}} & J\left(z_{0}, \bar{x}_{t-N}^{\epsilon}, \bar{I}_{t}\right):=\mu\left\|z_{0}-\bar{x}_{t-N}^{\epsilon}\right\|^{2}+\left\|\bar{y}_{t}-h\left(z_{N}\right)\right\|^{2}+\sum_{k=0}^{N-1}\left\|y_{t-N+k}-h\left(z_{k}\right)\right\|^{2}(4 \mathrm{a}) \\
\text { s.t. } & z_{k+1}=f\left(z_{k}, u_{t-N+k}\right), \quad k=0, \ldots, N-1 \\
& z_{k} \in \mathcal{X}, \quad k=0, \ldots, N .
\end{array}
$$

Using the solution of this problem $\hat{x}_{t-N}^{o}\left(\bar{I}_{t}\right)$, we construct an on-line correction formula of the form

$$
\hat{x}_{t-N}^{\epsilon}\left(I_{t}\right)=\hat{x}_{t-N}^{o}\left(\bar{I}_{t}\right)+\mathbf{K}_{t}^{o}\left(I_{t}-\bar{I}_{t}\right) .
$$

With this, we can compute a fast on-line estimate as soon as the true measurement $y_{t}$ becomes available. Here, $\mathbf{K}_{t}^{o}$ is a gain matrix that can be constructed by using NLP sensitivity and the Karush-Kuhn-Tucker matrix of the MHE problem evaluated at the solution of the background MHE problem (see the next section). In [24], it has been shown that this matrix is a Kalman-like gain matrix. The approximate state generated by the correction step at time $t$ is denoted as $\hat{x}_{t-N}^{\epsilon}\left(I_{t}\right)$. This has an associated cost $J\left(\hat{x}_{t-N}^{\epsilon}\left(I_{t}\right), \bar{x}_{t-N}^{\epsilon}, I_{t}\right)$ and error,

$$
\epsilon_{t}:=J\left(\hat{x}_{t-N}^{\epsilon}\left(I_{t}\right), \bar{x}_{t-N}^{\epsilon}, I_{t}\right)-J\left(\hat{x}_{t-N}^{o}\left(I_{t}\right), \bar{x}_{t-N}^{o}, I_{t}\right) .
$$


The current estimation error is $e_{t-N}^{\epsilon}:=\hat{x}_{t-N}^{\epsilon}-x_{t-N}$. At the next time step, the reference state is updated as $\bar{x}_{t-N+1}^{\epsilon} \leftarrow f\left(\hat{x}_{t-N}^{\epsilon}, u_{t-N}\right)$. In the following, we will refer to this algorithm as the approximate MHE algorithm.

The approximate MHE strategy can significantly reduce the on-line solution time because the expensive computational tasks are performed between sampling times while the correction step (5) can be computed almost instantaneously [10, 24]. The sensitivity computation is at least two orders of magnitude faster than the full NLP solution in $[24,21,23]$. However, a problem that arises in approximate MHE schemes is that the correction step introduces a numerical approximation error $\epsilon_{t}$ that is propagated at each time step through the reference initial state. In the next sections, we investigate under which conditions we can guarantee stability even in the presence of these numerical errors. In addition, we analyze the interplay between the system properties, numerical approximation errors and the dynamics of the estimation error $e_{t}^{\epsilon}$.

\section{Observability and Sensitivity Properties}

To start the discussion, we use the following assumptions and definitions.

Assumption 1 (System Properties)

- The sets $\Xi, \mathcal{H}$ and $U$ are compact.

- Any initial condition $x_{0}$ and control sequence $u_{t}, t \geq 0$ are such that, for any possible disturbance sequences $\xi_{t}, t \geq 0$, and $\eta_{t}, t \geq 0$, the system trajectory $x_{t}, t \geq 0$, lies in a closed and compact set $\mathcal{X}$.

- The functions $f$ and $h$ are $C^{2}$ functions with respect to both arguments $x \in \mathcal{X}$ and $u \in \mathcal{U}$. The associated Lipschitz constants are $k_{f}$ and $k_{h}$, respectively.

Definition 1 A continuous function $\varphi: \Re \rightarrow \Re$ is a $\mathcal{K}$ function if $\varphi(0)=0, \varphi(s)>0, \forall s>$ 0 and it is strictly increasing.

For the sake of simplicity, we will consider a representation of the MHE problem (4) of the form

$$
\min _{z_{0}} J\left(z_{0}, \bar{x}_{t-N}, I_{t}\right):=\mu\left\|z_{0}-\bar{x}_{t-N}\right\|^{2}+\left\|I_{t}^{y}-F\left(z_{0}, I_{t}^{u}\right)\right\|^{2}
$$

The nonlinear mapping $F: \Re^{n_{x}} \times \Re^{N n_{u}} \rightarrow \Re^{(N+1) n_{y}}$ has the structure

$$
F\left(z_{0}, I_{t}^{u}\right):=\left[\begin{array}{c}
h\left(z_{0}\right) \\
h \circ f^{u_{t-N}}\left(z_{0}\right) \\
\vdots \\
h \circ f^{u_{t-1}} \circ \ldots \circ f^{u_{t-N}}\left(z_{0}\right)
\end{array}\right] .
$$

To simplify the analysis, we will also make the following assumption: 
Assumption 2 (Constraints) The constraints $z_{k} \in \mathcal{X}, k=0, \ldots, N$ in (4) can be satisfied implicitly, and the optimal estimates never lie at the boundary of the set $\mathcal{X}$.

Later we will discuss extensions needed in order to relax this assumption. We emphasize that formulation (7) is only conceptual. Because of computational efficiency reasons, problem (4) should be solved in practice. We now impose requirements on the observability properties of the nonlinear discrete-time system (1) and relate them to the properties of (7).

Definition 2 (Observability Definition) [1, 19, 2] The system (1) is said to be observable in $N+1$ steps if there exists a $\mathcal{K}$-function $\varphi(\cdot)$ such that

$$
\left\|F\left(z_{0}, I^{u}\right)-F\left(z_{0}^{\prime}, I^{u}\right)\right\|^{2} \geq \varphi\left(\left\|z_{0}-z_{0}^{\prime}\right\|^{2}\right), \quad \forall z_{0}, z_{0}^{\prime} \in \mathcal{X}, \forall u \in \mathcal{U} .
$$

Assumption 3 (Observability Assumption)

System (1) is observable in $N+1$ steps $\forall z_{0} \in \mathcal{X}$ and $\forall u \in \mathcal{U}$.

This assumption implies that $\forall z_{0}, z_{0}^{\prime} \in \mathcal{X}$ and $\forall u \in \mathcal{U}, \exists \delta>0$ such that

$$
\left\|F\left(z_{0}^{\prime}, I^{u}\right)-F\left(z_{0}, I^{u}\right)\right\|^{2} \geq \delta\left\|z_{0}^{\prime}-z_{0}\right\|^{2} .
$$

In other words, Assumption 3 guarantees that different initial states give rise to distinguishable output trajectories. This also implies that the system

$$
I^{y}=F\left(z_{0}, I^{u}\right), \quad \forall z_{0} \in \mathcal{X}, \forall u, \in \mathcal{U}
$$

always has a unique solution. An issue related to Definition 2 is that it can be difficult to verify and quantify in practice (e.g., for large-scale nonlinear systems and in the presence of constraints). Motivated by this, we seek to relate the observability properties of the system to the numerical properties of the NLP problem because this can be verified automatically using a NLP solver [21]. Note that uniqueness of (11) is equivalent to guaranteeing that the unregularized $(\mu=0)$ MHE problem (7) has a unique solution. In optimization literature it is said that the NLP has a strict isolated minimizer. A strict isolated minimizer satisfies the so-called strong second-order sufficient conditions (SSOC), which we present here in the context of problem (7).

Lemma 1 (SSOC Conditions) [18] Let $J\left(z_{0}, \bar{x}, I\right)$ be a $C^{2}$ function w.r.t. $z_{0}$ in a neighborhood of $z_{0}^{o}$. Under Assumption 2, if $\varphi_{z}^{o}:=\nabla_{z} J\left(z_{0}^{o}, \bar{x}, I\right)=0$ and $w^{T} \mathcal{H}^{o} w>0$ with $\mathcal{H}^{o}:=\nabla_{z, z} J\left(z_{0}^{o}, \bar{x}, I\right)$ hold $\forall w$, then $z_{0}^{o}$ is a strict isolated minimizer.

The requirement of $J\left(z_{0}, \bar{x}, I\right)$ being a $C^{2}$ function follows from Assumption 1 . The above lemma can be modified to account explicitly for active constraints at the solution. In such a case, the vector $w$ needs to be restricted to the subspace of the variables at the boundary of $\mathcal{X}$. The analysis of this case would require a detailed structural analysis of the mapping $F(\cdot, \cdot)$ and of the set $\mathcal{X}$. Therefore, this analysis is omitted here. A detailed SSOC analysis in the context of MHE can be found in Chapters 3 and 6 in [20]. 
The gradient and the Hessian matrix evaluated at the solution $z_{0}^{o}$ are given by

$$
\begin{aligned}
\nabla_{z} J\left(z_{0}^{o}, \bar{x}, I\right) & =2 \mu\left(z_{0}^{o}-\bar{x}\right)-2 \frac{\partial F^{T}}{\partial z_{0}}\left(I^{y}-F\left(z_{0}^{o}, I^{u}\right)\right) \\
\nabla_{z, z} J\left(z_{0}^{o}, \bar{x}, I\right) & =2 \mu \mathbb{I}_{n_{x}}+2 \frac{\partial F^{T}}{\partial z_{0}} \frac{\partial F}{\partial z_{0}}-2 \frac{\partial^{2} F}{\partial z_{0}^{2}}\left(I^{y}-F\left(z_{0}^{o}, I^{u}\right)\right),
\end{aligned}
$$

where $\frac{\partial F}{\partial z_{0}}$ and ${\frac{\partial F}{\partial z_{0}}}^{T} \frac{\partial F}{\partial z_{0}}$ are the so-called observability and Grammian matrices, respectively. If we apply a second-order Taylor series expansion of the objective function around $z_{0}^{o}$ satisfying SSOC, we have

$$
\begin{aligned}
J\left(z_{0}^{\prime}, \bar{x}, I\right)-J\left(z_{0}^{o}, \bar{x}, I\right) & =\frac{1}{2}\left(z_{0}^{\prime}-z_{0}^{o}\right)^{T} \mathcal{H}^{o}\left(z_{0}^{\prime}-z_{0}^{o}\right) \\
& \geq \frac{1}{2} \lambda_{\min }\left(\mathcal{H}^{o}\right)\left\|z_{0}^{\prime}-z_{0}^{o}\right\|^{2}
\end{aligned}
$$

where $\lambda_{\min }\left(\mathcal{H}^{o}\right)>0$ if SSOC holds (see Figure 1). From (12a) it is not difficult to see that, for the special case in which $\mu=0$ and the residuals $I^{y}-F\left(z_{0}^{o}, I^{u}\right)$ vanish at the solution, solving problem (7) is equivalent to solving (11). The estimation strategy presented in [16] is based on the solution of this algebraic system. Note also that, in this special case, expression (13) reduces to (10) with $\delta=\frac{1}{2} \lambda_{\min }\left(\mathcal{H}^{o}\right)$. Moreover, the Hessian reduces to $\mathcal{H}^{o}=2{\frac{\partial F}{\partial z_{0}}}^{T} \frac{\partial F}{\partial z_{0}}$. Therefore, SSOC implies that the observability matrix is full rank and that the Grammian is positive definite. This was also noticed in $[15,17,14]$ in the context of linear systems. From this sequence of implications it is clear that the satisfaction of SSOC is a valid and general observability qualification. Observability properties have been traditionally analyzed a priori by using, for instance, a singular-value-decomposition (SVD) of the Grammian matrix at nominal state and control values. In nonlinear systems, however, it is well known that the system properties can change drastically with the nominal values. In addition, the numerical properties of the Grammian matrix can be related to Definition 2 only if the system model and output mappings are linear. Finally, computing the derivatives of the mapping $F(\cdot, \cdot)$ and performing the SVD decomposition can become expensive or cumbersome in large systems. The SSOC property, on the other hand, can be checked for complex MHE problems through modern NLP solvers and modeling capabilities [20]. This check can be performed a posteriori by solving the estimation problem. This can be useful, for instance, if the available measurements are noisy or if constraints are present.

Remark: To check for the observability qualification through SSOC we require the regularization term to be zero $(\mu=0)$. If the system is already observable, in the sense of Definition 2, setting $\mu>0$ will introduce a bias in the solution (i.e., this acts as a Tikhonov regularization) [4]. If the system is not observable, setting $\mu$ to a sufficient large value adds artificial curvature to the cost function, inducing a unique (but biased) solution. This regularization term can also be added internally by some NLP algorithms (e.g., a la Levenberg-Marquardt) [18] as the search proceeds.

The satisfaction of SSOC also has implications on the sensitivity of the solution to perturbations on the problem data $I$ around a reference solution $z_{0}^{o}(\bar{I})[3]$. To explore this, we use the following well-known result, adapted to the context of problem (7). 


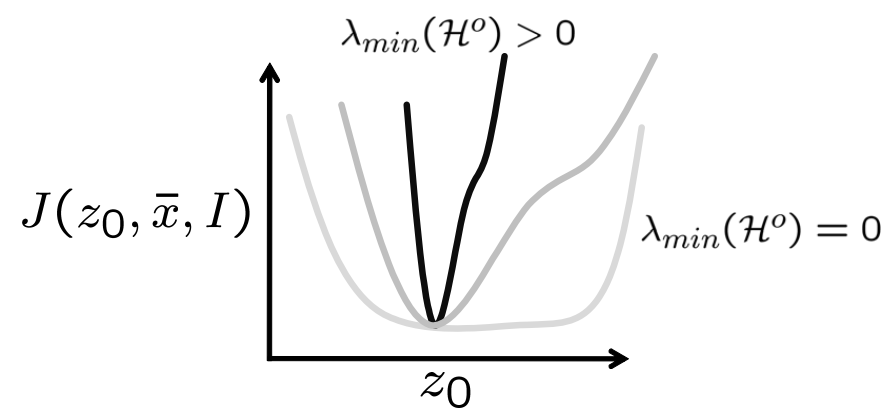

Figure 1: Sketch of curvature of cost function for observable $\left(\lambda_{\min }>0\right)$ and unobservable system $\left(\lambda_{\min }=0\right)$.

Theorem 2 (NLP Sensitivity) [8, 7]. If a nominal solution $z_{0}^{O}(\bar{I})$ satisfies SSOC, then the following hold:

- For I in a neighborhood of $\bar{I}$ there exists a unique, continuous and differentiable vector function $z_{0}^{o}(I)$ that is a strict isolated minimizer satisfying SSOC.

- The optimal cost is locally Lipschitz in a neighborhood of $\bar{I}$.

From these results, we can apply the implicit function theorem to (12a) at $z_{0}^{o}(\bar{I})$ to obtain a correction formula (5) with

$$
\mathbf{K}_{t}^{o}=\left.\frac{\partial z_{0}^{o}}{\partial I}\right|_{z_{0}^{o}(\bar{I}), \bar{x}, \bar{I}}=\mathcal{H}^{o-1} \varphi_{I}^{o}
$$

and $\varphi_{I}^{o}:=-\nabla_{z, I} J\left(z_{0}^{o}(\bar{I}), \bar{x}, \bar{I}\right)$. The sensitivity matrix can be bounded as

$$
\left\|\left.\frac{\partial z_{0}^{o}}{\partial I}\right|_{z_{0}^{o}(\bar{I}), \bar{x}, \bar{I}}\right\| \leq \frac{1}{\lambda_{\min }\left(\mathcal{H}^{o}\right)}\left\|\varphi_{I}^{o}\right\| .
$$

If the solution satisfies SSOC (system is observable), the solution is numerically stable to perturbations on $I$. This is reflected by a small sensitivity matrix. If, on the other hand, the Hessian becomes singular (system becomes unobservable), the sensitivity matrix grows unboundedly. This singularity represents a bifurcation point in the $z_{0}^{o}(I)-I$ space at which solutions of the MHE problem move from a set of minimizers to a set of saddle points or maximizers [11]. This is sketched in Figure 2.

Using the sensitivity results, we can now establish a rigorous bound on the error generated by the correction step of the approximate MHE algorithm.

Lemma 3 (Numerical Error Bound) Assume $z_{0}^{o}\left(\bar{I}_{t}\right)$ is a solution of (7) satisfying SSOC. Then, for $I_{t}$ in the neighborhood of $\bar{I}_{t}, \exists k_{x}, k_{J}, \epsilon_{t} \geq 0$, such that

$$
\begin{aligned}
\left\|\hat{x}_{t-N}^{\epsilon}\left(I_{t}\right)-\hat{x}_{t-N}^{o}\left(I_{t}\right)\right\| & \leq k_{x}\left\|I_{t}-\bar{I}_{t}\right\|^{2} \\
\epsilon_{t}=J\left(\hat{x}_{t-N}^{\epsilon}\left(I_{t}\right), \bar{x}_{t-N}^{\epsilon}, I_{t}\right)-J\left(\hat{x}_{t-N}^{o}\left(I_{t}\right), \bar{x}_{t-N}^{\epsilon}, I_{t}\right) & \leq k_{J}\left\|I_{t}-\bar{I}_{t}\right\|^{2} .
\end{aligned}
$$




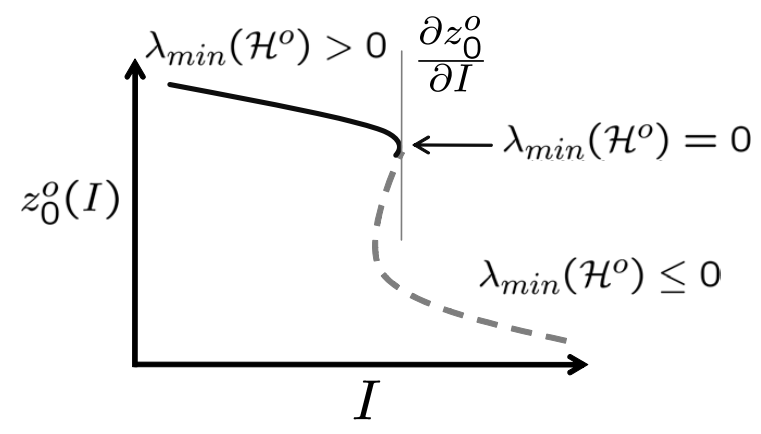

Figure 2: Sketch of path of minimizers $z_{0}^{o}\left(I_{t}\right)$ and presence of singular point (loss of observability).

Proof: We note that $\hat{x}_{t-N}^{o}\left(I_{t}\right)=z_{0}^{o}\left(I_{t}\right)$. Bound (16a) follows from Taylor's theorem [6, 22 ], while (16b) follows from (16a) and the Lipschitz continuity of the cost, as stated in Theorem 2. The error $\epsilon_{t}$ is always nonnegative because, if $\hat{x}_{t-N}^{o}\left(I_{t}\right)$ satisfies SSOC, then $J\left(\hat{x}_{t-N}^{o}\left(I_{t}\right), \bar{x}_{t-N}^{\epsilon}, I_{t}\right) \leq J\left(\hat{x}_{t-N}^{\epsilon}\left(I_{t}\right), \bar{x}_{t-N}^{\epsilon}, I_{t}\right)$.

\section{$4 \quad$ Stability Properties}

We now establish stability conditions for the estimation error of the approximate MHE algorithm. We define uniform bounds for the disturbances, the initial estimation error $e_{0}^{o}:=\hat{x}_{0}^{o}-x_{0}$ at $t=0$, and for the constant $\delta$ in the observability condition (10):

$$
r_{\xi}:=\max _{\xi_{t} \in \Xi}\left\|\xi_{t}\right\|, \quad r_{\eta}:=\max _{\eta_{t} \in \mathcal{H}}\left\|\eta_{t}\right\|, \quad d_{x}:=\max _{x_{0}, \hat{x}_{0}^{o} \in \mathcal{X}}\left\|\hat{x}_{0}^{o}-x_{0}\right\|, \quad \delta_{\min }:=\min _{\delta>0}\left\|\delta_{t}\right\| .
$$

To establish a reference for the stability conditions of the approximate algorithm, we revisit the stability results of [2] for the optimal MHE algorithm.

Theorem 4 (Stability of Optimal MHE Algorithm) If Assumptions 1 and 3 hold, then the optimal cost $J\left(\hat{x}_{t-N}^{o}, \bar{x}_{t-N}^{o}, \bar{I}_{t}\right)$ obtained from the solution of (3) can be bounded as

$$
\begin{aligned}
& J\left(\hat{x}_{t-N}^{o}, \bar{x}_{t-N}^{o}, I_{t}\right) \leq \mu\left\|x_{t-N}-\bar{x}_{t-N}^{o}\right\|^{2}+c^{2} \\
& J\left(\hat{x}_{t-N}^{o}, \bar{x}_{t-N}^{o}, I_{t}\right) \geq \frac{1}{2} \mu\left\|e_{t-N}^{o}\right\|^{2}+\frac{1}{2} \varphi\left(\left\|e_{t-N}^{o}\right\|^{2}\right)-\mu\left\|x_{t-N}-\bar{x}_{t-N}^{o}\right\|^{2}-c^{2} .
\end{aligned}
$$

Furthermore, the estimator error $e_{t-N}^{o}$ can be bounded as

$$
\left\|e_{t-N}^{o}\right\|^{2} \leq \zeta_{t-N},
$$


where $\zeta_{t-N}$ is generated by the sequence

$$
\begin{aligned}
\zeta_{0} & =\beta_{0} \\
\zeta_{t} & =\alpha \zeta_{t-1}+\beta \\
\alpha & =\frac{4}{\mu+\delta_{\min }}\left(2 \mu k_{f}^{2}\right) \\
\beta_{0} & =\frac{4}{\mu+\delta_{\text {min }}}\left(2 \mu d_{x}^{2}+c^{2}\right) \\
\beta & =\frac{4}{\mu+\delta_{\text {min }}}\left(2 \mu r_{\xi}^{2}+c^{2}\right) .
\end{aligned}
$$

If $\mu$ is selected such that $\alpha<1$, then as $t \rightarrow \infty$, we have $\left\|e_{\infty}^{o}\right\|^{2} \rightarrow \frac{\beta}{1-\alpha}$.

Proof: The complete proof of this theorem has been presented in [2]. A summary is given in Appendix A.

Theorem 4 does not make use of Assumption 2. The theorem states that, for a suitable choice of $\mu$, the estimation error sequence is convergent. Condition $\alpha<1$ becomes easier to satisfy as $\delta_{\min }$ increases (better observability). For $\delta_{\min }=0$ this condition can be satisfied only if $8 k_{f}^{2}<1$ ( $\mu$ cannot be used to control the error). The error diverges for $\delta_{\min } \leq-\mu$. This divergence illustrates the role of the regularization or arrival cost term in the cost function. The stability conditions are easier to satisfy with smaller $k_{f}$ (the more contractive the system). We should emphasize that, because of the nonlinearity of the system, the stability results should be used to analyze trends and effects of the system properties and of the parameters $\mu$ and $N$, rather than to guide design decisions.

The previous stability results require the MHE problem to be solved on-line to optimality. We now establish stability conditions for the approximate MHE algorithm. In particular, we analyze the propagation of $\epsilon_{t}$ through the estimator error sequence.

Theorem 5 (Stability of Approximate MHE Algorithm) If Assumptions 1 and 3, and the bounds of Lemma 3 hold, then the approximate cost $J\left(\hat{x}_{t-N}^{\epsilon}, \bar{x}_{t-N}^{\epsilon}, \bar{I}_{t}\right)$ can be bounded as

$$
\begin{aligned}
& J\left(\hat{x}_{t-N}^{\epsilon}, \bar{x}_{t-N}^{\epsilon}, I_{t}\right) \leq \mu\left\|x_{t-N}-\bar{x}_{t-N}^{\epsilon}\right\|^{2}+c^{2}+\epsilon_{t} \\
& J\left(\hat{x}_{t-N}^{\epsilon}, \bar{x}_{t-N}^{\epsilon}, I_{t}\right) \geq \frac{1}{2} \mu\left\|e_{t-N}^{\epsilon}\right\|^{2}+\frac{1}{2} \varphi\left(\left\|e_{t-N}^{\epsilon}\right\|^{2}\right)-\mu\left\|x_{t-N}-\bar{x}_{t-N}^{\epsilon}\right\|^{2}-c^{2} .
\end{aligned}
$$

Furthermore, the estimator error $e_{t-N}^{\epsilon}$ can be bounded as

$$
\left\|e_{t-N}^{\epsilon}\right\|^{2} \leq \bar{\zeta}_{t-N}
$$

where $\bar{\zeta}_{t-N}$ is generated by the sequence

$$
\begin{aligned}
\bar{\zeta}_{0} & =\bar{\beta}_{0} \\
\bar{\zeta}_{t} & =\bar{\alpha} \bar{\zeta}_{t-1}+\bar{\beta} \\
\bar{\beta}_{0} & =\beta_{0}+\frac{4}{\mu+\delta_{\text {min }}}\left(\kappa_{2} r_{\xi}^{2}+\kappa_{3} r_{\eta}^{2}\right) \\
\bar{\beta} & =\beta+\frac{4}{\mu+\delta_{\text {min }}}\left(\kappa_{2} r_{\xi}^{2}+\kappa_{3} r_{\eta}^{2}\right) \\
\bar{\alpha} & =\alpha+\frac{4 \kappa_{1}}{\mu+\delta_{\text {min }}}
\end{aligned}
$$


with $\kappa_{1}, \kappa_{2}$ and $\kappa_{3}$ defined in Appendix $C$. If $\mu$ is selected such that $\bar{\alpha}<1$, then as $t \rightarrow \infty$, we have $\left\|e_{\infty}^{\epsilon}\right\|^{2} \rightarrow \frac{\bar{\beta}}{1-\bar{\alpha}}$.

Proof: See Appendix B.

Corollary 6 (Asymptotic Behavior in Absence of Disturbances) If Assumptions 1 and 3, the bounds of Lemma 3, and $r_{\xi}=r_{\eta}=0$ hold, then $\left\|e_{t-N}^{o}\right\|^{2}$ and $\left\|e_{t-N}^{\epsilon}\right\|^{2}$ converge exponentially to zero as

$$
\begin{aligned}
\left\|e_{t-N}^{o}\right\|^{2} & \leq \alpha^{t-N} \beta_{0} \\
\left\|e_{t-N}^{\epsilon}\right\|^{2} & \leq\left(\alpha+\frac{4 \kappa_{1}}{\mu+\delta_{\min }}\right)^{t-N} \beta_{0} .
\end{aligned}
$$

Proof: In the absence of noise disturbances, we have that $\bar{\beta}=\beta=0$ and $\bar{\beta}_{0}=\beta_{0}$. The result follows.

From Corollary 6 we see that the rate of convergence of the approximate estimator is $4 \kappa_{1} /\left(\mu+\delta_{\text {min }}\right)$ slower than that of the optimal counterpart. An unexpected result from this analysis is the fact that, even in the absence of noise disturbances, the rate of convergence of the approximate MHE algorithm is not the same as that of the optimal counterpart. The reason is that the estimation error $\left\|e_{t-N}^{\epsilon}\right\|^{2}$ is always propagated to the predicted output $\bar{y}_{t+1}$ generating an error $\epsilon_{t+1}$ at the next step. For instance, if we have a large initial estimation error (bad initial reference state $\bar{x}_{0}$ ), then $\left\|e_{0}^{\epsilon}\right\|^{2}$ will be large and will tend to give larger approximation errors during the first time steps. On the other hand, this also implies that, as soon as $\left\|e_{t-N}^{\epsilon}\right\|^{2}=0$, the predicted and true output measurements coincide and $\epsilon_{t+1}=0$ for all subsequent $t$.

In the presence of noise disturbances, from (19) and (21) it is clear that additional errors are introduced by the correction step. From Appendix $\mathrm{C}$ we see that the additional errors are always multiplied by $k_{J}$. That is, as expected, the stability properties of the estimators coincide if $\epsilon_{t}=0$. This happens, for instance, if the model and output mappings are linear, since the MHE problem reduces to a quadratic programming problem and the correction step is exact.

The bounds of Lemma 3 are related to the observability properties of the system and on the numerical stability properties of the MHE problem. Therefore, these bounds are problem dependent. However, from (15) and (5) it is clear that $\epsilon_{t}$ tends to zero as $\lambda_{\min }\left(\mathcal{H}^{o}\right)$ shifts away from zero. This means that, as the numerical stability properties of the problem improve, the correction step (5) will not generate appreciable changes in the state estimate. This desired effect can be influenced by increasing the horizon length or by increasing the regularization term $\mu$, since this tends to increase the curvature of the solution, which is reflected in $\lambda_{\min }\left(\mathcal{H}^{o}\right)$. This behavior can also be appreciated from term $4 \kappa_{1} /\left(\mu+\delta_{\text {min }}\right)$, which tends to decrease as $\delta_{\min }$ and $\mu$ increase.

\section{$5 \quad$ Numerical Case Studies}

In this section, we illustrate the effect of the system properties and of numerical errors on the performance of the optimal and approximate MHE estimators. In addition, we analyze some of the the stability conditions developed in the previous sections. 


\subsection{Effect of System Properties}

We consider an MHE scenario of the system:

$$
\begin{aligned}
\frac{d x_{1}}{d \tau} & =x_{2}(\tau) \\
\frac{d x_{2}}{d \tau} & =x_{1}(\tau)+x_{2}(\tau)+\left(1-x_{1}(\tau)^{2}\right) x_{3}(\tau)+u(\tau) \\
\frac{d x_{3}}{d \tau} & =-x_{1}(\tau)+x_{3}(\tau) .
\end{aligned}
$$

Here, the only measured state is assumed to be $x_{1}$. An analysis of the observability matrix of this system reveals that it becomes unobservable for $x_{1}=1$ and $x_{1}=-1$ [5]. The continuous-time model is transformed into a discrete-time form through an implicit Euler discretization scheme. We use $\bar{x}_{0}=\left[\begin{array}{lll}1 & 1 & 1\end{array}\right]$ as the initial state estimate and an estimation horizon $N=5$.

We run the optimal MHE estimator with noise-free measurements generated artificially and with no regularization $(\mu=0)$. In Figure 3 we illustrate the convergence of the estimator when the system is observable and unobservable. In the top graph we present the convergence to an observable state (the measured one) $x_{1}$. The estimator quickly converges to the true state. In the middle graph we see that the estimator converges to the true state $x_{3}$ when the estimator is observable. Around time step 80, however, $x_{1}=-1$, and the estimate of $x_{3}$ diverges. In the bottom graph we show the minimum eigenvalue of the Hessian matrix extracted from the NLP solver. Notice the small magnitude of the eigenvalue $\left(O\left(10^{-5}\right)\right)$ even in the first time steps when the system is observable. The system is weakly observable. At time step 80 there is a sudden jump of five orders of magnitude of the eigenvalue to $O\left(10^{-10}\right)$. The NLP solver is able to detect the lost observability.

In Figure 4 we illustrate the convergence of the estimator under the same conditions but with a regularization of $\mu=0.01$. Note that the estimator converges in the observable range more slowly. In addition, it diverges when $x_{1}$ gets close to -1 . This simulation reinforces the analysis of Theorem 4 where we see that, when the system becomes unobservable $\left(\delta_{\min }, \lambda_{\min } \rightarrow 0\right)$, the regularization parameter $\mu$ cannot be used to control the estimator error. This result is counterintuitive because it implies that, if the system is already observable and noise-free, the arrival cost degrades only the convergence of the estimator error. Nevertheless, we emphasize that, if one is interested in quantifying the uncertainty of the state estimates, then introducing the arrival cost with covariance information (as in the formulations used in $[19,24]$ ) is relevant because it summarizes information not included in the estimation window. As expected, we have also seen that when the system is observable, the regularization term helps to counteract the effects of noise.

\subsection{Effect of Numerical Errors}

We now consider a MHE scenario on the nonlinear continuous stirred tank reactor [12]:

$$
\begin{aligned}
\frac{d x_{1}}{d \tau} & =\frac{x_{1}(\tau)-1}{\theta}+k_{0} \cdot x_{1}(\tau) \cdot \exp \left[\frac{-E_{a}}{x_{2}(\tau)}\right] \\
\frac{d x_{2}}{d \tau} & =\frac{x_{2}(\tau)-x_{2_{f}}}{\theta}-k_{0} \cdot x^{1}(\tau) \cdot \exp \left[\frac{-E_{a}}{x_{2}(\tau)}\right]+\alpha \cdot u(\tau) \cdot\left(x_{2}(\tau)-x_{2_{c w}}\right) .
\end{aligned}
$$



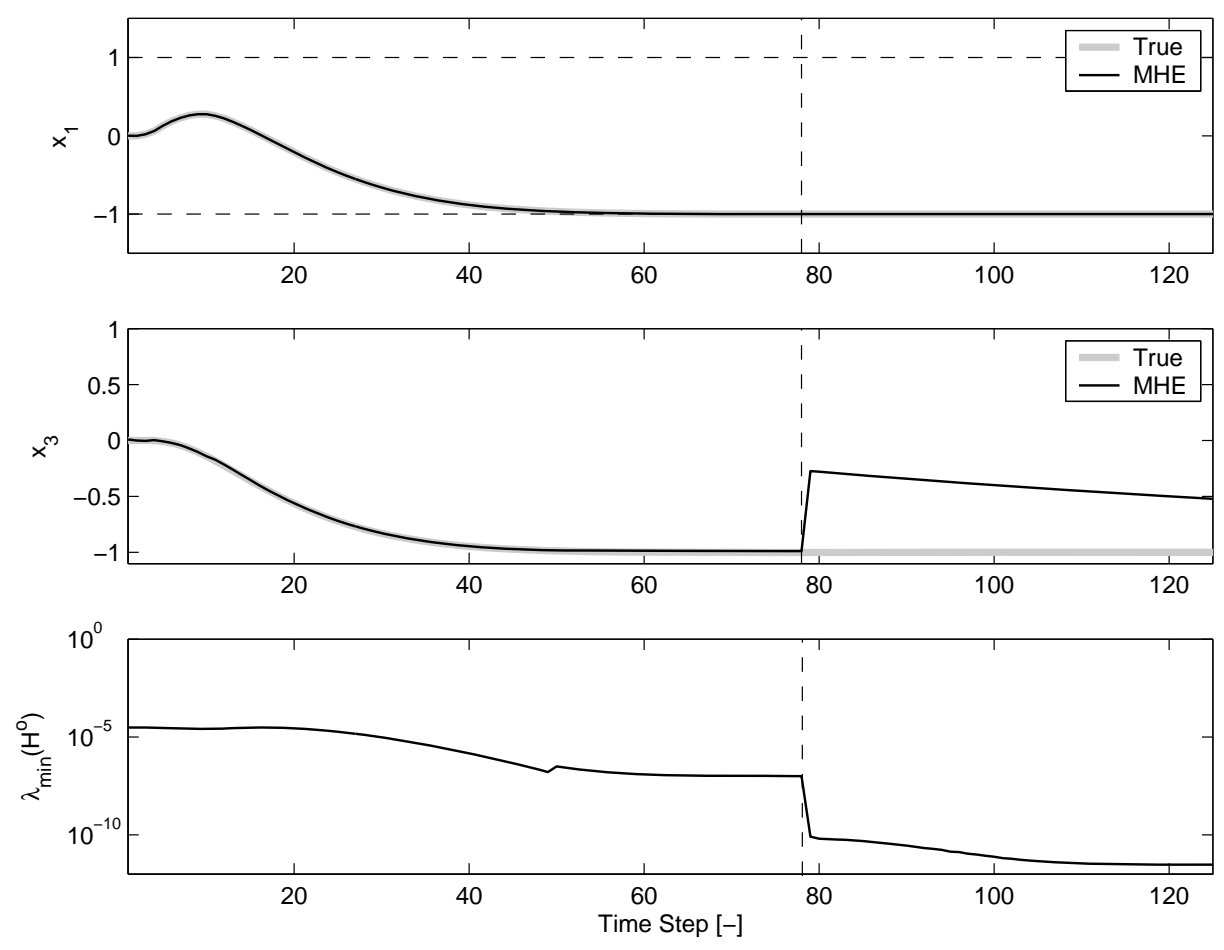

Figure 3: Effect of observability on convergence of optimal MHE estimator in the absence of disturbances and $\mu=0$. The system becomes unobservable when $x_{1}=-1$.

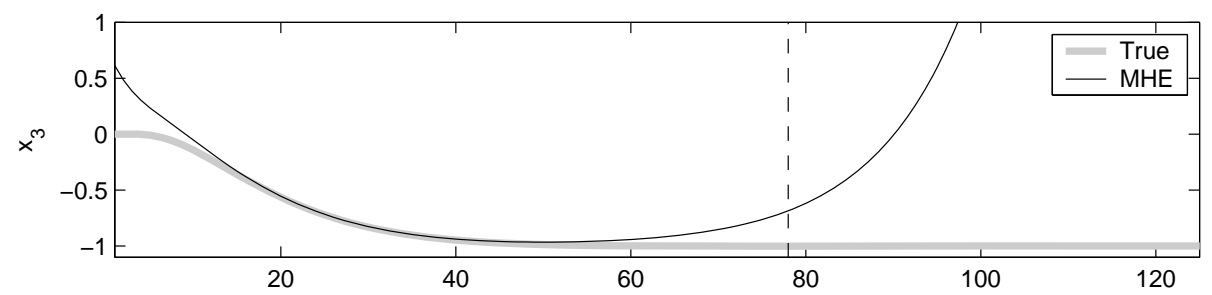

Figure 4: Effect of observability on convergence of optimal MHE estimator in the absence of disturbances and $\mu=0.1$.

The system involves two states $x=\left[x_{1}, x_{2}\right]$ corresponding to the concentration and temperature, respectively, and one control $u$ corresponding to the cooling water flowrate. The continuous-time model is transformed into a discrete-time form through an implicit Euler discretization scheme. The temperature is used as the measured output $\left(y_{t}=x_{2_{t}}\right)$ to infer the concentration. The model parameters are $x_{2_{c w}}=0.38, x_{2_{f}}=0.395, E_{a}=5$, $\alpha=1.95 \times 10^{4}$, and $k_{0}=300$. We use batch data generated from a simulated closed-loop feedback control scenario. The simulated states are corrupted with different levels of Gaussian noise with variance $\sigma^{2}$ measured as a percentage relative to the nominal temperature value. The corrupted temperature values are used to study the effect of noise disturbances $\eta_{t}$. We use $\bar{x}_{0}=\left[\begin{array}{ll}0.15 & 0.15\end{array}\right]$ as the initial reference state, a regularization penalty $\mu=30$, 

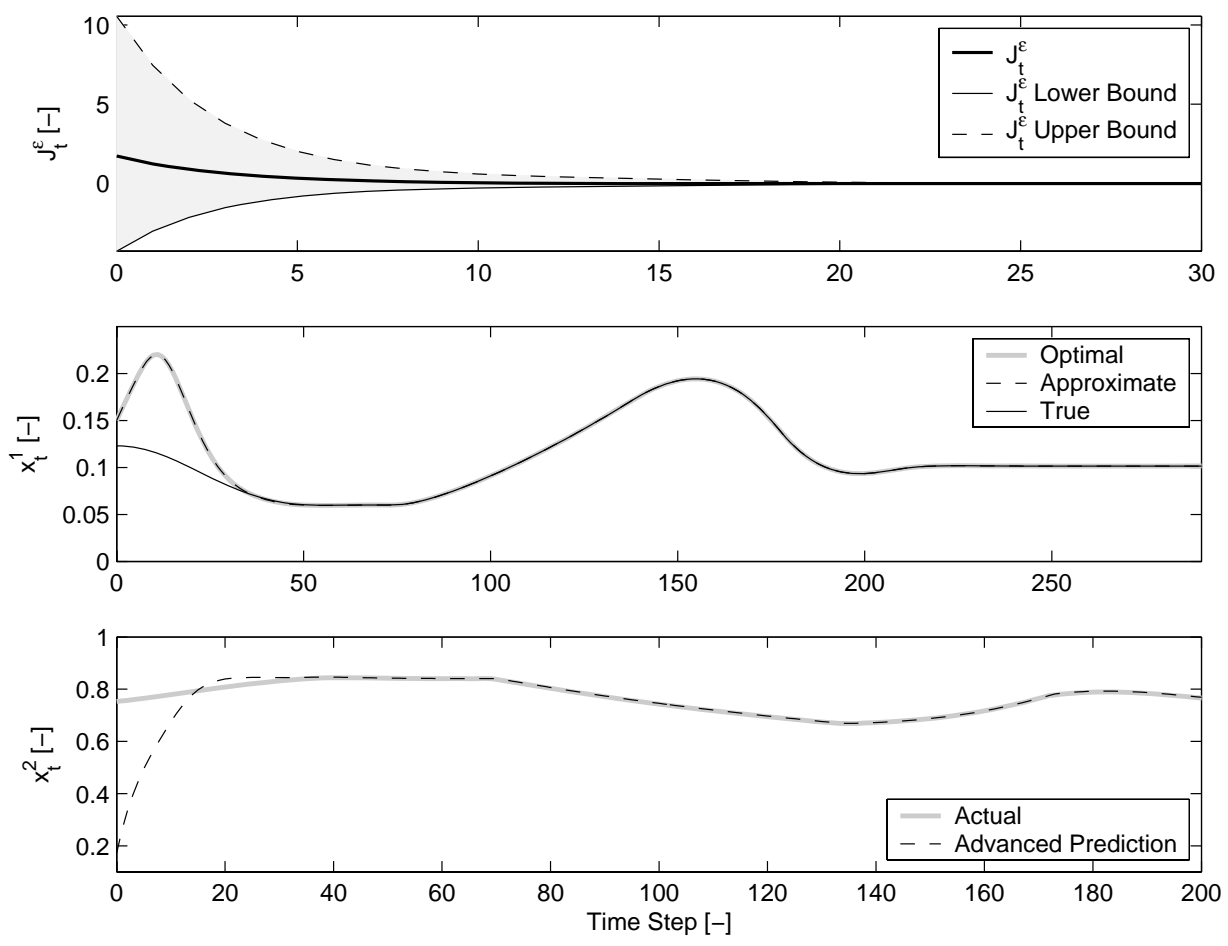

Figure 5: Convergence of approximate and optimal MHE estimator in the absence of disturbances.

and an estimation horizon $N=5$.

In Figure 5 we compare the performance of both the optimal and approximate MHE algorithms in the absence of disturbances $\left(\sigma^{2}=0 \%\right)$. In the top graph, we present the approximate cost $J_{t}^{\epsilon}:=J\left(\hat{x}_{t-N}^{\epsilon}, \bar{x}_{t-N}^{\epsilon}, I_{t}\right)$ and its corresponding upper and lower bounds. These bounds correspond to the right-hand sides of equations (33) and (34), respectively. The estimator recovers from the bad initial reference (bound $d_{x}$ in Theorem 5), and the estimator error converges to the origin in about 30 time steps (reflected as a zero cost). In the middle graph, we contrast the trajectories of the inferred state for the optimal and approximate estimators, while in the bottom graph we contrast the predicted $\bar{y}_{t}$ and true measurements $y_{t}$. These generate the perturbation $\left\|y_{t}-\bar{y}_{t}\right\|^{2}$ in (16) for the approximate estimator. As can be seen, even if the initial prior is far away from the true state, both estimators exhibit the same performance. The contraction of the estimation errors is quite fast, implying that constants $\alpha, \bar{\alpha}$ are quite small. This also implies that the approximation errors $\epsilon_{t}$ are negligible.

In Figure 6 we compare the performance of the estimators in the presence of noise disturbances with $\sigma=2.5 \%$. In the top graph, we present the approximate cost and corresponding upper and lower bounds in which we can see that the estimator converges to a neighborhood of the origin. In the middle graph, we see that the trajectories of the inferred state for both estimators are still very close to each other. In the bottom graph, we present 

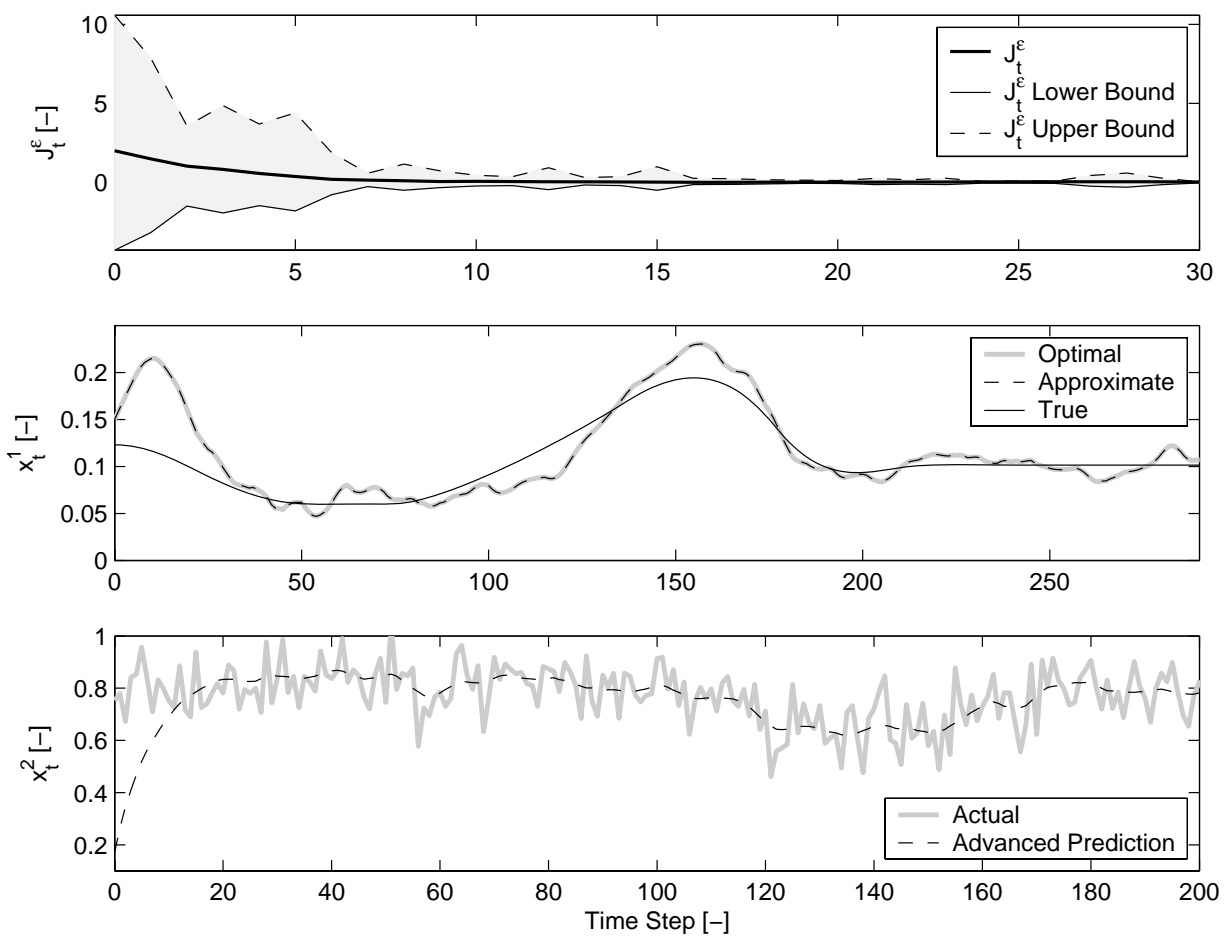

Figure 6: Convergence of approximate and optimal MHE estimator in the presence of disturbances.

the relatively large perturbations $\left\|y_{t}-\bar{y}_{t}\right\|^{2}$ reflected by larger deviations between the predicted and true temperatures compared to those observed in the noise-free scenario.

In the top graph of Figure 7 we present the total sum of the approximation errors $\epsilon_{\text {sum }}=\sum_{t} \epsilon_{t}$ over the whole horizon for scenarios with increasing levels of noise $\sigma^{2}=$ $[0 \%, 2.5 \%, 5 \%, 7.5 \%, 10 \%]$. As expected, the approximation errors tend to increase with the noise level. Nevertheless, their overall magnitude remains small $O\left(10^{-5}\right)$. This is mainly due to the strong observability properties of the system. To illustrate this, in the bottom graph we present trends of $\lambda_{\min }\left(\mathcal{H}^{o}\right)$ for MHE problems with different horizons and two different regularization terms. As can be seen, for the $\mu=0$ case, the system is strongly observable even for very short horizons $\left(\lambda_{\min }\left(\mathcal{H}^{o}\right)>0\right)$. This value also increases with the horizon length, as expected. Note also that, by setting $\mu=1$, the eigenvalues are shifted away from zero. From bound (15), it can be seen that this decreases the sensitivity of the state estimates and thus reduce the approximation errors as it is observed in the simulation results.

\section{Conclusions and Future Work}

In this work, we have studied the stability properties of an approximate algorithm for moving horizon estimation (MHE). The algorithm is particularly suitable for large-scale 

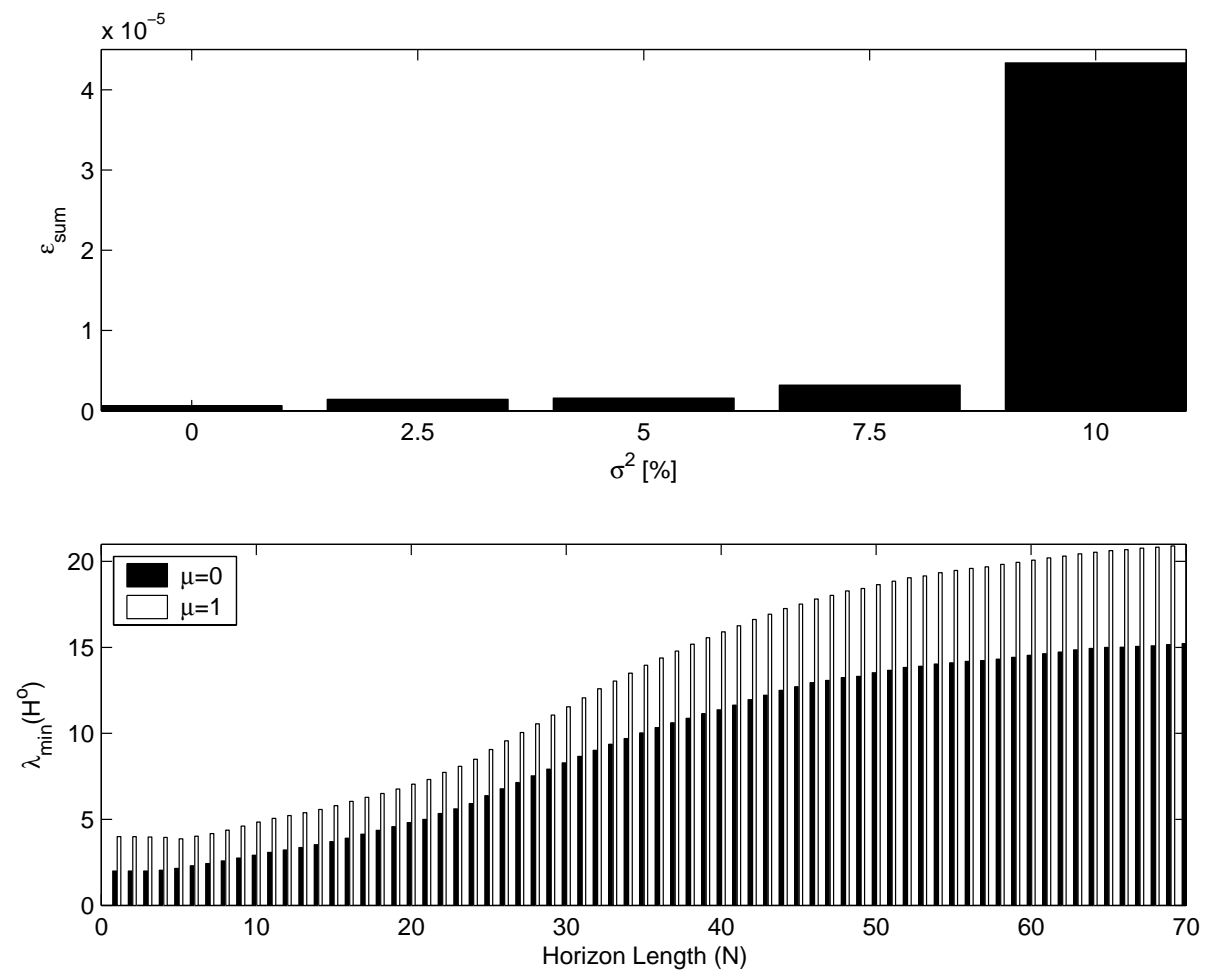

Figure 7: Effect of noise on approximation errors (top). Effect of horizon length and regularization on $\lambda_{\min }\left(\mathcal{H}^{o}\right)$ (bottom).

systems because it can significantly reduce the on-line solution time by constructing fast approximations using reference solutions computed in between sampling times. The stability analysis reveals that the estimation error converges at a similar rate compared to that of an optimal MHE counterpart. In addition, the observability properties of the nonlinear system have a strong impact on the convergence of the estimator error. This insight has been used to derive guidelines able to reduce the impact of numerical errors.

As part of future work, we are interested in considering the more general MHE formulation presented in [19]. We recognize that, for strong disturbances or ill-conditioned problems, the corrected state $\hat{x}_{t}^{\epsilon}\left(I_{t}\right)$ in (5) can become a worse approximation than the uncorrected state $x^{o}\left(\bar{I}_{t}\right)$ if error bounds of Lemma 3 do not hold. We are thus interested in developing strategies able to preserve stability.

\section{Acknowledgments}

This work was supported by the U.S. Department of Energy through contract DE-AC0206CH11357. We acknowledge the insightful comments and suggestions of the reviewers. 


\section{A Proof of Theorem 4}

To construct the estimator error sequence $e_{t-N}^{o}$, we obtain lower and upper bounds for the cost function $J\left(\hat{x}_{t-N}^{o}, \bar{x}_{t-N}^{o}, I_{t}\right)$. An upper bound can be obtained by noticing that, since $\hat{x}_{t-N}^{o}$ is optimal, it gives a smaller cost than the true state $x_{t-N}$ (because of the regularization term). With this,

$$
\begin{aligned}
J\left(\hat{x}_{t-N}^{o}, \bar{x}_{t-N}^{o}, I_{t}\right) & \leq J\left(x_{t-N}, \bar{x}_{t-N}^{o}, I_{t}\right) \\
& =\mu\left\|x_{t-N}-\bar{x}_{t-N}^{o}\right\|+\left\|I_{t}^{y}-F\left(x_{t-N}, I_{t}^{u}\right)\right\|^{2} .
\end{aligned}
$$

As shown in equation (32) in [2], the second term on the right-hand side can be bounded by using the disturbance bounds $r_{\xi}$ and $r_{\eta}$. These terms can be lumped into a constant $c^{2}$ to give

$$
J\left(\hat{x}_{t-N}^{o}, \bar{x}_{t-N}^{o}, I_{t}\right) \leq \mu\left\|x_{t-N}-\bar{x}_{t-N}^{o}\right\|+c^{2} .
$$

To construct a lower bound, we start from the optimal cost,

$$
J\left(\hat{x}_{t-N}^{o}, \bar{x}_{t-N}^{o}, I_{t}\right)=\mu\left\|\hat{x}_{t-N}^{o}-\bar{x}_{t-N}^{o}\right\|+\left\|I_{t}^{y}-F\left(\hat{x}_{t-N}^{o}, I_{t}^{u}\right)\right\|^{2} .
$$

The second term can be bounded from

$$
\begin{aligned}
\left\|F\left(x_{t-N}, I_{t}^{u}\right)-F\left(\hat{x}_{t-N}^{o}, I_{t}^{u}\right)\right\|^{2} & =\left\|\left(I_{t}^{y}-F\left(\hat{x}_{t-N}^{o}, I_{t}^{u}\right)\right)+\left(F\left(x_{t-N}, I_{t}^{u}\right)-I_{t}^{y}\right)\right\|^{2} \\
& \leq 2\left\|I_{t}^{y}-F\left(\hat{x}_{t-N}^{o}, I_{t}^{u}\right)\right\|^{2}+2 c^{2}
\end{aligned}
$$

so that

$$
\begin{aligned}
\left\|I_{t}^{y}-F\left(\hat{x}_{t-N}^{o}, I_{t}^{u}\right)\right\|^{2} & \geq \frac{1}{2}\left\|F\left(x_{t-N}, I_{t}^{u}\right)-F\left(\hat{x}_{t-N}^{o}, I_{t}^{u}\right)\right\|^{2}-c^{2} \\
& \geq \frac{1}{2} \varphi\left(\left\|x_{t-N}-\hat{x}_{t-N}^{o}\right\|^{2}\right)-c^{2} .
\end{aligned}
$$

The last inequality arises from the Observability Assumption 3. We now bound the first term in (26) from

$$
\begin{aligned}
& \mu\left\|x_{t-N}-\hat{x}_{t-N}^{o}\right\| \leq 2 \mu\left\|x_{t-N}-\bar{x}_{t-N}^{o}\right\|+2 \mu\left\|\hat{x}_{t-N}^{o}-\bar{x}_{t-N}^{o}\right\| \\
& \mu\left\|\hat{x}_{t-N}^{o}-\bar{x}_{t-N}^{o}\right\| \geq \frac{1}{2} \mu\left\|x_{t-N}-\hat{x}_{t-N}^{o}\right\|-\mu\left\|x_{t-N}-\bar{x}_{t-N}^{o}\right\| .
\end{aligned}
$$

Merging terms and using (10) with $\delta_{m i n}$, we obtain

$$
J\left(\hat{x}_{t-N}^{o}, \bar{x}_{t-N}^{o}, I_{t}\right) \geq \frac{1}{2} \mu\left\|e_{t-N}^{o}\right\|+\frac{1}{2} \delta_{\min }\left\|e_{t-N}^{o}\right\|^{2}-\mu\left\|x_{t-N}-\bar{x}_{t-N}^{o}\right\|-c^{2} .
$$

Combining bounds (26) and (30) yields

$$
\begin{aligned}
\frac{1}{2} \mu\left\|e_{t-N}^{o}\right\|+\frac{1}{2} \delta_{\text {min }}\left\|e_{t-N}^{o}\right\|^{2} & \leq 2 \mu\left\|x_{t-N}-\bar{x}_{t-N}^{o}\right\|+2 c^{2} \\
\left\|e_{t-N}^{o}\right\|^{2} & \leq \frac{4 \mu}{\mu+\delta_{\text {min }}}\left\|x_{t-N}-\bar{x}_{t-N}^{o}\right\|^{2}+\frac{4}{\mu+\delta_{\text {min }}} c^{2} .
\end{aligned}
$$


The proof is completed by relating the current estimation error to the previous estimation error as

$$
\begin{aligned}
\left\|x_{t-N}-\bar{x}_{t-N}^{o}\right\|^{2} & =\left\|f\left(x_{t-N-1}, u_{t-N-1}\right)+\xi_{t-N-1}-f\left(\hat{x}_{t-N-1}^{o}, u_{t-N-1}\right)\right\|^{2} \\
& \leq 2 k_{f}^{2}\left\|x_{t-N-1}-\hat{x}_{t-N-1}^{o}\right\|^{2}+2\left\|\xi_{t-N-1}\right\|^{2} \\
& =2 k_{f}^{2}\left\|e_{t-N-1}^{o}\right\|^{2}+2\left\|\xi_{t-N-1}\right\|^{2} .
\end{aligned}
$$

The convergent sequence follows from (31) and (32). The proof is complete.

\section{B Proof of Theorem 5}

A lower bound for the approximate cost $J\left(\hat{x}_{t-N}^{\epsilon}, \bar{x}_{t-N}^{\epsilon}, I_{t}\right)$ can be obtained as in Appendix A to give

$$
J\left(\hat{x}_{t-N}^{\epsilon}, \bar{x}_{t-N}^{\epsilon}, I_{t}\right) \geq \frac{1}{2} \mu\left\|e_{t-N}^{\epsilon}\right\|^{2}+\frac{1}{2} \delta_{\min }\left(\left\|e_{t-N}^{\epsilon}\right\|^{2}\right)-\mu\left\|x_{t-N}-\bar{x}_{t-N}^{\epsilon}\right\|^{2}-c^{2} .
$$

As an upper bound we consider

$$
J\left(\hat{x}_{t-N}^{\epsilon}, \bar{x}_{t-N}^{\epsilon}, I_{t}\right)=J\left(\hat{x}_{t-N}^{o}, \bar{x}_{t-N}^{\epsilon}, I_{t}\right)+\epsilon_{t} .
$$

With this,

$$
J\left(\hat{x}_{t-N}^{\epsilon}, \bar{x}_{t-N}^{\epsilon}, I_{t}\right) \leq \mu\left\|x_{t-N}-\bar{x}_{t-N}^{\epsilon}\right\|^{2}+c^{2}+\epsilon_{t} .
$$

Combining the upper and lower bounds, we have

$$
\begin{aligned}
\mu\left\|x_{t-N}-\bar{x}_{t-N}^{\epsilon}\right\|^{2}+c^{2}+\epsilon_{t} & \geq \frac{1}{2} \mu\left\|e_{t-N}^{\epsilon}\right\|^{2}+\frac{1}{2} \delta_{\min }\left(\left\|e_{t-N}^{\epsilon}\right\|^{2}\right)-\mu\left\|x_{t-N}-\bar{x}_{t-N}^{\epsilon}\right\|^{2}-c^{2} \\
\left\|e_{t-N}^{\epsilon}\right\|^{2} & \leq \frac{4 \mu}{\mu+\delta_{\min }}\left\|x_{t-N}-\bar{x}_{t-N}^{\epsilon}\right\|^{2}+\frac{4}{\mu+\delta_{\min }} c^{2}+\frac{2}{\mu+\delta_{\min }} \epsilon_{t} .
\end{aligned}
$$

The first term on the right-hand side is bounded as

$$
\left\|x_{t-N}-\bar{x}_{t-N}^{\epsilon}\right\|^{2} \leq 2 k_{f}^{2}\left\|e_{t-N-1}^{\epsilon}\right\|+2 r_{\xi}^{2} .
$$

To bound $\epsilon_{t}$, we first note that the only element changing from $\bar{I}_{t}$ is the predicted measurement $\bar{y}_{t}$ which is computed from extrapolation of the previous state estimate $\hat{x}_{t-1}^{\epsilon}$. With this, (16) reduces to

$$
\epsilon_{t} \leq k_{J}\left\|\bar{y}_{t}-y_{t}\right\|^{2} .
$$

The error between measurements is bounded as

$$
\begin{aligned}
\left\|\bar{y}_{t}-y_{t}\right\|^{2} & =\left\|h\left(f\left(\hat{x}_{t-1}^{\epsilon}, u_{t-1}\right)\right)-h\left(f\left(x_{t-1}, u_{t-1}\right)+\xi_{t-1}\right)+\eta_{t-1}\right\|^{2} \\
& \leq 2 k_{h}^{2}\left(2 k_{f}^{2}\left\|e_{t-1}^{\epsilon}\right\|^{2}+2\left\|\xi_{t-1}\right\|\right)+2\left\|\eta_{t-1}\right\|^{2} \\
& \leq 4 k_{h}^{2} k_{f}^{2}\left\|e_{t-1}^{\epsilon}\right\|^{2}+4 k_{h}^{2} r_{\xi}^{2}+2 r_{\eta}^{2}
\end{aligned}
$$


where $e_{t-1}^{\epsilon}$ is the estimation error of the state $\hat{x}_{t-1}^{\epsilon}$ obtained from propagation of the initial state $\hat{x}_{t-N-1}^{\epsilon}$. As a consequence, this error can be related to the the desired estimation error $e_{t-N-1}^{\epsilon}$ through backpropagation:

$$
\begin{aligned}
\left\|e_{t-1}^{\epsilon}\right\|^{2} & =\left\|\hat{x}_{t-1}^{\epsilon}-x_{t-1}\right\|^{2} \\
& =\left\|f\left(\hat{x}_{t-2}^{\epsilon}, u_{t-2}\right)-f\left(x_{t-2}, u_{t-2}\right)-\xi_{t-2}\right\|^{2} \\
& \leq 2 k_{f}^{2}\left\|e_{t-2}^{\epsilon}\right\|^{2}+2\left\|\xi_{t-2}\right\|^{2} \\
& \leq 2 k_{f}^{2}\left\|f\left(\hat{x}_{t-3}^{\epsilon}, u_{t-3}\right)-f\left(x_{t-3}, u_{t-3}\right)+\xi_{t-3}\right\|^{2}+2\left\|\xi_{t-2}\right\|^{2} \\
& \vdots \\
& \leq\left(2 k_{f}^{2}\right)^{N}\left\|e_{t-N-1}^{\epsilon}\right\|^{2}+\sum_{k=0}^{N-1}\left(2 k_{f}^{2}\right)^{k} r_{\xi}^{2} \\
& =\left(2 k_{f}^{2}\right)^{N}\left\|e_{t-N-1}^{\epsilon}\right\|^{2}+\frac{\left(2 k_{f}^{2}\right)^{N}-1}{2 k_{f}^{2}-1} r_{\xi}^{2}
\end{aligned}
$$

With this,

$$
\begin{aligned}
\left\|\bar{y}_{t}-y_{t}\right\|^{2} & \leq 4 k_{h}^{2} k_{f}^{2}\left\|e_{t-1}^{\epsilon}\right\|^{2}+4 k_{h}^{2} r_{\xi}^{2}+2 r_{\eta}^{2} \\
& \leq 4 k_{h}^{2} k_{f}^{2}\left(\left(2 k_{f}^{2}\right)^{N}\left\|e_{t-N-1}^{\epsilon}\right\|^{2}+\frac{\left(2 k_{f}^{2}\right)^{N}-1}{2 k_{f}^{2}-1} r_{\xi}^{2}\right)+4 k_{h}^{2} r_{\xi}^{2}+2 r_{\eta}^{2} \\
& \leq 2 k_{h}^{2}\left(2 k_{f}^{2}\right)^{N+1}\left\|e_{t-N-1}^{\epsilon}\right\|^{2}+2 k_{h}^{2}\left(2+\frac{\left(2 k_{f}^{2}\right)^{N+1}-1}{2 k_{f}^{2}-1}\right) r_{\xi}^{2}+2 r_{\eta}^{2} .
\end{aligned}
$$

We have thus obtained the required bound for $\epsilon_{t}$ :

$$
\epsilon_{t} \leq 2 \kappa_{1}\left\|e_{t-N-1}^{\epsilon}\right\|^{2}+2 \kappa_{2} r_{\xi}^{2}+2 \kappa_{3} r_{\eta}^{2} .
$$

Substituting (43) and (37) in (36), we have

$$
\begin{gathered}
\left\|e_{t-N}^{\epsilon}\right\|^{2} \leq \frac{4 \mu}{\mu+\delta_{\min }}\left(2 k_{f}^{2}\left\|e_{t-N-1}^{\epsilon}\right\|^{2}+2 r_{\xi}^{2}\right)+\frac{4}{\mu+\delta_{\min }} c^{2} \\
+\frac{2}{\mu+\delta_{\min }}\left(2 \kappa_{1}\left\|e_{t-N-1}^{\epsilon}\right\|^{2}+2 \kappa_{2} r_{\xi}^{2}+2 \kappa_{3} r_{\eta}^{2}\right) \\
=\left(\frac{8 k_{f}^{2} \mu}{\mu+\delta_{\min }}+\frac{4 \kappa_{1}}{\mu+\delta_{\min }}\right)\left\|e_{t-N-1}^{\epsilon}\right\|^{2}+\frac{4}{\mu+\delta_{\min }}\left(2 \mu r_{\xi}^{2}+c^{2}\right) \\
+\frac{4}{\mu+\delta_{\min }}\left(\kappa_{2} r_{\xi}^{2}+\kappa_{3} r_{\eta}^{2}\right)
\end{gathered}
$$


where $\kappa_{1}, \kappa_{2}$, and $\kappa_{3}$ are defined in Appendix C. The error sequence follows:

$$
\begin{aligned}
\left\|e_{t-N}^{\epsilon}\right\|^{2} & \leq \bar{\zeta}_{t-N} \\
\bar{\zeta}_{0} & =\bar{\beta}_{0} \\
\bar{\zeta}_{t} & =\bar{\alpha} \bar{\zeta}_{t-1}+\bar{\beta} \\
\bar{\beta}_{0} & =\beta_{0}+\frac{4}{\mu+\delta_{\min }}\left(\kappa_{2} r_{\xi}^{2}+\kappa_{3} r_{\eta}^{2}\right) \\
\bar{\beta} & =\beta+\frac{4}{\mu+\delta_{\min }}\left(\kappa_{2} r_{\xi}^{2}+\kappa_{3} r_{\eta}^{2}\right) \\
\bar{\alpha} & =\alpha+\frac{4 \kappa_{1}}{\mu+\delta_{\min }} .
\end{aligned}
$$

The proof is complete.

\section{Constants}

$$
\begin{aligned}
\kappa_{1} & =k_{J} k_{h}^{2}\left(2 k_{f}^{2}\right)^{N+1} \\
\kappa_{2} & =k_{J} k_{h}^{2}\left(2+\frac{\left(2 k_{f}^{2}\right)^{N+1}-1}{2 k_{f}^{2}-1}\right) \\
\kappa_{3} & =k_{J} .
\end{aligned}
$$

\section{References}

[1] M. Alamir. Optimization based nonlinear observers revisited. Int. Journal of Control, 72(13):1204-1217, 1999.

[2] A. Alessandri, M. Baglietto, and G. Battistelli. Moving-horizon state estimation for nonlinear discrete-time systems: New stability results and approximation schemes. Automatica, 44:1753-1765, 2008.

[3] S. Basu and Y. Bresler. The stability of nonlinear least squares problems and the cramer-rao bound. IEEE Transactions on Signal Processing, 48:3426-3436, 2000.

[4] L. Blank. State estimation analysed as an inverse problem. In Assessment and Future Directions of NMPC, pages 335-346, 2007.

[5] C. Boehm, Rolf Findeisen, and Frank Allgoewer. Avoidance of Poorly Observable Trajectories: A predictive control perspective. In Proceedings of the 17th IFAC World Congress, Seoul, Korea, pages 1952-1957, 2008.

[6] J. E. Dennis and R. B. Schnabel. Numerical Methods for Unconstrained Optimization and Nonlinear Equations. SIAM, Philadelphia, PA, 1996.

[7] A. V. Fiacco. Sensitivity analysis for nonlinear programming using penalty methods. Math. Programm., 10:287-311, 1976. 
[8] A. V. Fiacco. Introduction to Sensitivity and Stability Analysis in Nonlinear Programming. Academic Press, New York, 1983.

[9] R. Findeisen and F. Allgöwer. Computational delay in nonlinear model predictive control. Proc. Int. Symp. Adv. Control of Chemical Processes ADCHEM 2003, Hong Kong, 2004.

[10] R. Findeisen, M. Diehl, T. Burner, F. Allgöwer, H. G. Bock, and J. P. Schlöder. Efficient output feedback nonlinear model predictive control. In Proceedings of American Control Conference, pages 4752-4757, 2002.

[11] J. Guddat, F. Guerra Vazquez, and H. T. Jongen. Parametric Optimization: singularities, pathfollowing and jumps. BG Teubner, 1990.

[12] G. A. Hicks and W. H. Ray. Approximation methods for optimal control synthesis. Can. J. Chem. Eng., 40:522-529, 1971.

[13] A. H. Jaswinski. Stochastic Processes and Filtering Theory. Academic Press, 1970.

[14] S. S. Keerthi and E. G. Gilbert. Optimal infinite-horizon feedback laws for general class of constrained discrete-time systems: Stability and moving-horizon approximations. IEEE Trans. Auto. Cont., 57(2):265-293, 1988.

[15] H. Michalska and D. Q. Mayne. Moving horizon observers and observer-based control. IEEE Trans. Autom. Control, 40(6):995-1006, 1995.

[16] P. E. Moraal and J. W. Grizzle. Observer design for nonlinear systems with discretetime systems. IEEE Trans. Autom. Control, 40(3):395-404, 1995.

[17] K. Muske and T. F. Edgar. Nonlinear state estimation. In Nonlinear Process Control, pages 311-370, 1997.

[18] J. Nocedal and S. Wright. Numerical Optimization. Springer, New York, NY, 1999.

[19] C. V. Rao, J. B. Rawlings, and D. Q. Mayne. Constrained state estimation for nonlinear discrete-time systems: Stability and moving horizon approximations. IEEE Trans. Autom. Control, 48(2):246-258, 2003.

[20] V. M. Zavala. Computational Strategies for the Operation of Large-Scale Chemical Processes, Ph. D. thesis. Carnegie Mellon University, 2008.

[21] V. M. Zavala and L. T. Biegler. Optimization-based strategies for the operation of low-density polyethylene tubular reactors: Moving horizon estimation. Computers and Chemical Engineering, 33(1):379-390, 2008.

[22] V. M. Zavala and L. T. Biegler. The advanced step nmpc controller: Stability, optimality and robustness. Automatica, 45:86-93, 2009.

[23] V. M. Zavala and L. T. Biegler. Nonlinear programming strategies for state estimation and model predictive control. In Nonlinear Model Predictive Control, pages 419-432, 2009. 
[24] V. M. Zavala, C. D. Laird, and L. T. Biegler. A fast moving horizon estimation algorithm based on nonlinear programming sensitivity. Journal of Process Control, 18(9):876-884, 2008.

(To be removed before publication) The submitted manuscript has been created by the University of Chicago as Operator of Argonne National Laboratory ("Argonne") under Contract No. DE-AC02-06CH11357 with the U.S. Department of Energy. The U.S. Government retains for itself, and others acting on its behalf, a paid-up, nonexclusive, irrevocable worldwide license in said article to reproduce, prepare derivative works, distribute copies to the public, and perform publicly and display publicly, by or on behalf of the Government. 